\title{
TRATAMIENTO PSICOLÓGICO EN MUJERES VÍCTIMAS DE VIOLENCIA CONYUGAL
}

\author{
LUZ Clarita FÉlix-MONTES \\ Reynalda Gavilán-Centeno \\ Claudia Ríos-Cataño \\ https://orcid.org/0000-0001-7547-9093 \\ Universidad Continental, Huancayo, Perú \\ Correo electrónico: Luzfelixmontes@gmail.com \\ Recibido: 21 de agosto del 2020 / Aceptado: 24 de septiembre del 2020 \\ doi: https://doi.org/10.26439/persona2020.n023(2).4829
}

\begin{abstract}
Resumen. El estudio tuvo como objetivo identificar los avances terapéuticos, el grado de efectividad y las principales manifestaciones clínicas en mujeres víctimas de violencia conyugal a través de la revisión de literatura. Se hizo una búsqueda en cinco bibliotecas virtuales: EBSCOhost, Scielo, Science Direct, Redalyc y ProQuest. Los criterios de inclusión fueron artículos científicos a partir del año 2000 en adelante, en español y en la población mundial. Se encontró que la terapia cognitivo-conductual (TCC), la terapia racional emotiva (TRE) y las terapias contextuales fueron las más utilizadas. Entre ellas, para la TCC existe mayor evidencia de desarrollo y efectividad en resultados, con técnicas empleadas según la manifestación clínica, que varían de 8 a 20 sesiones, con mayor frecuencia en formato individual. Por ello fue la más aplicada en tratamientos psicológicos para mujeres maltratadas, respaldada por su efectividad. Como nueva propuesta, la terapia contextual también resultó efectiva en la intervención, pero no hay evidencia de mayor empleabilidad. Las manifestaciones clínicas más trabajadas fueron la depresión, ansiedad, baja autoestima y trastorno de estrés postraumático (TEPT). Sin embargo, las limitaciones de este tipo de estudio no permiten llegar a conclusiones generalizables.
\end{abstract}

Palabras clave: violencia contra la mujer / eficacia / intervención psicológica 


\title{
PSYCHOLOGICAL TREATMENT IN FEMALE VICTIMS OF SPOUSAL VIOLENCE
}

\begin{abstract}
The study aimed to identify the therapeutic advances, degree of effectiveness and main clinical manifestations in female victims of spousal violence through literature review. Five online libraries were searched: EBSCOhost, SciELO, ScienceDirect, Redalyc and ProQuest. The inclusion criteria were scientific articles written in Spanish and published worldwide from the year 2000 onwards. It was found that the most commonly used therapies were cognitive behavioral therapy (CBT), rational emotive behavior therapy (REBT) and contextual therapy. Out of these, CBT results showed more evidence of development and effectiveness. The techniques were chosen according to the clinical manifestation, lasted from 8 to 20 sessions and were conducted more frequently on an individual basis. Thus, CBT was the most frequently used psychological treatment for battered women due to its efficacy. As a new proposal, contextual therapy was also effective in interventions, but there is no evidence of its widespread use. The most frequently studied clinical manifestations were depression, anxiety, low self-esteem and post-traumatic stress disorder (PTSD). However, this type of studies does not allow to draw generalizable conclusions.
\end{abstract}

Keywords: violence against women / efficacy / psychological intervention 


\section{INTRODUCCIÓN}

La Organización Mundial de la Salud (OMS, 2017) indica que el $35 \%$ de las mujeres en el mundo han sufrido violencia en algún momento de su vida; de ellas un $30 \%$ refiere ser víctima de maltrato por su pareja. Por su parte, la Organización Panamericana de la Salud (OPS, 2018) describe que en Sudamérica esta problemática puede afectar a casi el $60 \%$ de la población femenina. Uno de los últimos reportes de la OMS, el 7 de mayo del 2020, durante el confinamiento por la pandemia del coronavirus en Europa, señala que se registró un aumento de hasta un 60 \% de llamadas por mujeres víctimas de violencia.

La violencia de género es definida por la Organización de las Naciones Unidas (ONU, citado en Calvo González y Camacho Bejarano, 2014) como "cualquier acto o intención que origina daño o sufrimiento físico, sexual o psicológico a las mujeres, incluyendo las amenazas de dichos actos, la coerción o privación arbitraria de libertad, ya sea en la vida pública o privada". Es considerada como un problema de salud pública que se ha mantenido a lo largo de la historia afectando a muchas mujeres en el mundo (Calvo González y Camacho Bejarano, 2014) con secuelas que originan, a corto y largo plazo, problemas emocionales y trastornos mentales, como depresión, trastorno de estrés postraumático (TEPT), ansiedad y baja autoestima (Labrador et al., 2002).

Al respecto, la OMS sugiere priorizar la intervención desde ámbitos educativos, sociales y sanitarios (Calvo González y Camacho Bejarano, 2014). Por tanto, un aspecto importante para iniciar el tratamiento es el psicológico. El tratamiento se define como una intervención profesional, basada en métodos y técnicas psicológicas en un contexto clínico, donde el profesional busca eliminar o disminuir el sufrimiento de la persona en consulta, o instruir el fortalecimiento de habilidades para hacer frente a los diversos problemas que afronta, como la violencia conyugal (Labrador et al., 2002). Dadas estas características que trabaja el tratamiento psicológico, Romero (2010) plantea consideraciones que se deben tener en cuenta para lograr eficacia en la intervención con mujeres maltratadas: en primer lugar, la posición del terapeuta (juicios personales) no debe interferir en el compromiso para el trabajo con las víctimas de violencia, en el que se tendrá prioridad por la seguridad de la mujer y sus hijos; en segundo lugar, la propuesta de una intervención específica que dependa del caso, donde se genere un vínculo positivo, un interés genuino por el momento de su proceso personal y por el nivel de gravedad de sus secuelas, para evitar que vuelva a sentirse maltratada y cuestionada. Estas consideraciones pueden garantizar la calidad de las intervenciones y contribuir a la recuperación efectiva de la mujer atendida.

En esta línea, Cáceres-Ortiz, Labrador-Encinas, Ardila-Mantilla y Parada-Ortiz (2011), en su estudio sobre la efectividad del tratamiento psicológico cognitivo-conductual, lograron una mejora significativa de los síntomas: TEPT, depresión, ansiedad, inadaptación, así como aumento de la autoestima y disminución considerable de las 
cogniciones postraumáticas. Asimismo, Vaca-Ferrer, Ferro-García y Valero-Aguayo (2020), desde la intervención de terapias contextuales para las mujeres víctimas de violencia de género, consiguieron que un $62 \%$ de las mujeres que acudieron a más del $80 \%$ de la intervención mostraran una mejoría clínicamente significativa; y tras la terapia, las mujeres que obtuvieron puntuaciones de un malestar severo (ansiedad, depresión) se redujeron en un $23 \%$.

La importancia de esta revisión radica en presentar al profesional en psicología el uso de alguna práctica terapéutica que resulte más efectiva ante las secuelas psicológicas de la mujer maltratada, para que logre recuperar el control de su vida, aumente su seguridad y, por tanto, su autonomía, remedie el impacto psicológico y salga de manera definida del círculo de violencia. El objetivo de esta investigación fue identificar los avances terapéuticos, grado de efectividad y tratamiento para las principales manifestaciones clínicas en mujeres víctimas de violencia conyugal a través de la revisión de literatura.

\section{MÉTODO}

Se realizó una búsqueda bibliográfica en cinco bibliotecas virtuales: EBSCOhost, Scielo, Science Direct, Redalyc y ProQuest. Las palabras clave utilizadas fueron "violencia contra la mujer", "eficacia" e "intervención psicológica", y sus combinaciones. Los criterios de inclusión fueron estos: artículos científicos originales del año 2000 en adelante y en idioma español; se excluyeron resúmenes de congresos, revisiones, estudios de caso y monografías. Para el análisis de los datos se llevó a cabo un examen cualitativo, se elaboraron categorías y subcategorías de análisis.

\section{RESULTADOS Y DISCUSIÓN}

En la tabla 1, se indica autores, año de publicación, país de origen y base de datos de los 14 artículos de investigación originales utilizados. 
Tabla 1

Artículos encontrados en las bases de datos

\begin{tabular}{|c|c|c|c|}
\hline Autor(es) & Año & País & Base de datos \\
\hline Viñas Velásquez y Fernández de Juan & 2007 & España & EBSCOhost \\
\hline Labrador y Alonso & 2007 & México & Redalyc \\
\hline Bravo Campanón & 2008 & España & EBSCOhost \\
\hline $\begin{array}{l}\text { Labrador, Fernández Velasco } \\
\text { y Rincón González }\end{array}$ & 2009 & España & ProQuest \\
\hline Cáceres-Ortiz et al. & 2011 & Colombia & Scielo \\
\hline Iraizoz & 2012 & España & EBSCOhost \\
\hline $\begin{array}{l}\text { Madrigal, Cardenal, Téllez, } \\
\text { Ortiz-Tallo y Jiménez }\end{array}$ & 2012 & España & ProQuest \\
\hline $\begin{array}{l}\text { Sarasua, Zubizarreta, De Corral } \\
\text { y Echeburúa }\end{array}$ & 2013 & España & ProQuest \\
\hline Matud, Fortes y Medina & 2014 & España & Science Direct \\
\hline Santandreu y Ferrer-Pérez & 2014 & España & ProQuest \\
\hline $\begin{array}{l}\text { Habigzang, Schneider, Petroli Frizzo } \\
\text { y Pizarro de Freitas }\end{array}$ & 2018 & Brasil & EBSCOhost \\
\hline Jaramillo-Sierra y Ripoll-Núñez & 2018 & Colombia & ProQuest \\
\hline Habigzang, Gomes y Maciel & 2019 & Brasil & Scielo \\
\hline $\begin{array}{l}\text { Vaca-Ferrer, Ferro-García } \\
\text { y Valero-Aguayo }\end{array}$ & 2020 & España & ProQuest \\
\hline
\end{tabular}

Elaboración propia

\section{AVANCES TERAPÉUTICOS PARA LA INTERVENCIÓN A MUJERES MALTRATADAS}

La violencia de género es un problema social de mayor envergadura que tiene consecuencias psicoemocionales en la vida de la mujer, por lo que requiere de un abordaje sólido para menguar su reincidencia. En la tabla 2, se detallan las propuestas de intervención psicológica planteadas, las áreas trabajadas en cada una, el año de aplicación y el autor correspondiente. 
Tabla 2

Propuesta terapéutica y sus características individuales de abordaje por artículo

\begin{tabular}{|c|c|c|}
\hline Autor/año & Propuesta de intervención & Técnicas empleadas \\
\hline $\begin{array}{l}\text { Labrador y Alonso } \\
\text { (2007) }\end{array}$ & $\begin{array}{l}\text { Programa de intervención } \\
\text { breve y específico para } \\
\text { el tratamiento del TEPT, } \\
\text { diseñado por Labrador y } \\
\text { Rincón }\end{array}$ & $\begin{array}{l}\text { - } \quad \text { Terapia de exposición } \\
\text { - } \quad \text { Psicoeducación } \\
\text { - Entrenamiento en control de la respiración } \\
\text { - } \quad \text { Reestructuración cognitiva } \\
\text { - } \quad \text { Entrenamiento en solución de problemas } \\
\text { Formato: grupal ( } 43 \text { mujeres) } \\
\text { N. }{ }^{\circ} \text { de sesiones/tiempo: } 8 \text { (100 min c/u) } \\
\text { Medidas: pretratamiento, postratamiento y } \\
\text { seguimiento (1 y } 3 \text { meses) }\end{array}$ \\
\hline $\begin{array}{l}\text { Viñas Velásquez y } \\
\text { Fernández de Juan } \\
\text { (2007) }\end{array}$ & $\begin{array}{l}\text { Intervención a } \\
\text { mujeres maltratadas } \\
\text { en organizaciones } \\
\text { gubernamentales (OG) y no } \\
\text { gubernamentales (ONG) }\end{array}$ & $\begin{array}{l}\text { - Tratamiento con terapia breve } \\
\text { - Intervención en crisis (cuando era necesario) } \\
\text { - } \quad \text { Técnicas gestálticas, conductistas, humanistas } \\
\text { - } \quad \text { Entrenamiento en habilidades sociales, } \\
\text { autoestima, aumento de conciencia de la } \\
\text { situación } \\
\text { - Psicoeducación } \\
\text { OG: tiempo limitado en intervención (12 semanas) } \\
\text { ONG: tiempo de intervención necesario (aprox. } 2 \\
\text { años) }\end{array}$ \\
\hline $\begin{array}{l}\text { Bravo Campanón } \\
\text { (2008) }\end{array}$ & $\begin{array}{l}\text { Intervención con las } \\
\text { unidades familiares: Plan de } \\
\text { Atención Integral (PAI) }\end{array}$ & $\begin{array}{l}\text { - Autonomía personal y responsabilidad } \\
\text { - } \quad \text { Apoyo en las decisiones y acciones para } \\
\text { - } \quad \text { Apoyo en la interpretación de su rol como } \\
\text { mujer y de las consecuencias de los malos } \\
\text { tratos } \\
\text { Formato: individual (19 mujeres) } \\
\text { N.º de sesiones/tiempo: } 2 \text { años (quincenal) } \\
\text { Medidas: evaluación inicial, intervención, } \\
\text { seguimiento, finalización de intervención }\end{array}$ \\
\hline Labrador et al. (2009) & $\begin{array}{l}\text { Programa de tratamiento } \\
\text { de Labrador y Rincón } \\
\text { (2002): tratamiento breve e } \\
\text { individual del TEPT }\end{array}$ & $\begin{array}{l}\text { - } \quad \text { Psicoeducación } \\
\text { - } \quad \text { Entrenamiento en control de la activación } \\
\text { - } \quad \text { Terapia de exposición } \\
\text { - } \quad \text { Terapia cognitiva } \\
\text { Formato: individual ( } 20 \text { mujeres) } \\
\text { N. }{ }^{\circ} \text { de sesiones/tiempo: } 8 \text { (60 min c/u) } \\
\text { Medidas: pretratamiento, postratamiento, } \\
\text { seguimiento (6 meses) }\end{array}$ \\
\hline
\end{tabular}


(continuación)

\begin{tabular}{|c|c|c|}
\hline $\begin{array}{l}\text { Cáceres-Ortiz et al. } \\
\text { (2011) }\end{array}$ & $\begin{array}{l}\text { Tratamiento cognitivo- } \\
\text { conductual (guiado por el } \\
\text { programa de tratamiento de } \\
\text { Labrador y Rincón, 2002) }\end{array}$ & $\begin{array}{l}\text { - } \quad \text { Psicoeducación } \\
\text { - } \quad \text { Relajación } \\
\text { - } \quad \text { Práctica de actividades agradables } \\
\text { - } \quad \text { Entrenamiento asertivo y afrontamiento } \\
\text { - } \quad \text { Activación } \\
\text { - } \quad \text { Terapia cognitiva } \\
\text { - } \quad \text { Terapia de exposición } \\
\text { Formato: grupal (73 mujeres) } \\
\text { N. de sesiones/tiempo: } 8 \text { (100 a } 120 \text { min c/u) } \\
\text { Medidas: evaluación, tratamiento, seguimiento } \\
\text { (1, } 3 \text { y } 6 \text { meses) }\end{array}$ \\
\hline Iraizoz (2012) & $\begin{array}{l}\text { Terapia racional emotiva de } \\
\text { Ellis (para el trastorno de } \\
\text { ansiedad generalizada) }\end{array}$ & $\begin{array}{l}\text { - } \quad \text { Psicoeducación } \\
\text { - } \quad \text { Reestructuración cognitiva } \\
\text { - } \quad \text { Técnicas de autoinstrucción } \\
\text { - } \quad \text { Tiempo fuera } \\
\text { - } \quad \text { Relajación muscular progresiva de Jacobson } \\
\text { - } \quad \text { Habilidades sociales } \\
\text { - } \quad \text { Resolución de problemas } \\
\text { - } \quad \text { Tolerancia a la frustración } \\
\text { Formato: individual } \\
\text { Medidas: evaluación, tratamiento, finalización y } \\
\text { evaluación de los resultados }\end{array}$ \\
\hline $\begin{array}{l}\text { Madrigal et al. } \\
\text { (2012) }\end{array}$ & $\begin{array}{l}\text { Tratamiento psicológico } \\
\text { (bases de la TCC) }\end{array}$ & $\begin{array}{l}\text { - Intervención psicológica con fundamentación } \\
\text { - } \quad \text { Trabajo desde áreas de autoestima } \\
\text { - } \quad \text { Depresión } \\
\text { - } \quad \text { Ansiedad } \\
\text { - El nivel de inmunoglobulina A en saliva } \\
\text { Formato: grupal ( } 60 \text { mujeres) } \\
\text { N. }^{\circ} \text { de sesiones: } 12 \\
\text { Medidas: pretratamiento, tratamiento y } \\
\text { postratamiento }\end{array}$ \\
\hline Sarasua et al. (2013) & $\begin{array}{l}\text { Programa individual } \\
\text { cognitivo-conductual }\end{array}$ & $\begin{array}{l}\text { - Aspectos motivacionales, transmitir empatía y } \\
\text { - } \quad \text { Catarsis emocional } \\
\text { - } \quad \text { Psicoeducación } \\
\text { - } \quad \text { Reevaluación cognitiva } \\
\text { - } \quad \text { Entrenamiento en habilidades de } \\
\text { - } \quad \text { Relajación muscular progresiva } \\
\text { - } \quad \text { Técnicas de autoexposición } \\
\text { Formato: individual (121 mujeres) } \\
\text { N. }{ }^{\circ} \text { de sesiones/tiempo: } 12 \text { (60 min c/u por } 3 \\
\text { meses) } \\
\text { Medidas: evaluación, tratamiento, seguimiento (1, } \\
3,6 \text { y } 12 \text { meses) }\end{array}$ \\
\hline
\end{tabular}


(continuación)

\begin{tabular}{|c|c|c|}
\hline $\begin{array}{l}\text { Santandreu } \\
\text { y Ferrer-Pérez } \\
\text { (2014) }\end{array}$ & $\begin{array}{l}\text { Tratamiento cognitivo- } \\
\text { conductual }\end{array}$ & $\begin{array}{l}\text { - } \quad \text { Psicoeducación } \\
\text { - } \quad \text { Activación conductual } \\
\text { - } \quad \text { Técnicas de inervación vagal } \\
\text { - } \quad \text { Respiración diafragmática } \\
\text { - } \quad \text { Técnicas de exposición e imaginación en vivo } \\
\text { - } \quad \text { Reestructuración cognitiva } \\
\text { Formato: individual (129 mujeres) } \\
\text { N. }{ }^{\circ} \text { de sesiones/tiempo: } 12 \text { (50 min c/u) } \\
\text { Medidas: evaluación, tratamiento, seguimiento (3 } \\
\text { meses) }\end{array}$ \\
\hline Matud et al. (2014) & $\begin{array}{l}\text { Programa de tratamiento } \\
\text { psicológico individual, con } \\
\text { bases de la TCC }\end{array}$ & $\begin{array}{l}\text { - } \quad \text { Psicoeducación } \\
\text { - } \quad \text { Reestructuración cognitiva } \\
\text { - } \quad \text { Técnicas para mejorar la autoestima y } \\
\text { - } \quad \text { Fomentar la comunicación y las habilidades } \\
\text { - } \quad \text { Enciales } \\
\text { - } \quad \text { Prema de decisiones } \\
\text { Formato: individual ( } 128 \text { mujeres) } \\
\text { N. }{ }^{\circ} \text { de sesiones/tiempo: } 15 \text { a } 20 \text { ( } 90 \text { min c/u) } \\
\text { Medidas: evaluación, tratamiento, postratamiento }\end{array}$ \\
\hline $\begin{array}{l}\text { Habigzang et al. } \\
\text { (2018) }\end{array}$ & $\begin{array}{l}\text { Intervención cognitivo- } \\
\text { conductual individual }\end{array}$ & $\begin{array}{l}\text { - } \quad \text { Psicoeducación y reestructuración cognitiva } \\
\text { - } \quad \text { Exposición gradual a los recuerdos } \\
\text { - } \quad \text { Resolución de problemas } \\
\text { - } \quad \text { Prevención de recurrencia } \\
\text { Formato: individual (11 mujeres) } \\
\text { N. }{ }^{\circ} \text { de sesiones/tiempo: } 13 \text { (60 min c/u) } \\
\text { Medidas: evaluación, tratamiento, seguimiento }\end{array}$ \\
\hline $\begin{array}{l}\text { Jaramillo-Sierra y } \\
\text { Ripoll-Núñez (2018) }\end{array}$ & $\begin{array}{l}\text { Aproximación sistémica } \\
\text { (terapia breve centrada en } \\
\text { soluciones) }\end{array}$ & $\begin{array}{l}\text { - } \quad \text { Psicoeducación sobre violencia } \\
\text { - Elaboración de un plan de seguridad } \\
\text { - } \quad \text { Tiempo de descanso negociado } \\
\text { Formato: pareja (7) } \\
\text { N. }{ }^{\circ} \text { de sesiones/tiempo: } 6 \\
\text { Medidas: pretratamiento y postratamiento }\end{array}$ \\
\hline $\begin{array}{l}\text { Habigzang et al. } \\
\text { (2019) }\end{array}$ & $\begin{array}{l}\text { Terapia cognitivo- } \\
\text { conductual }\end{array}$ & 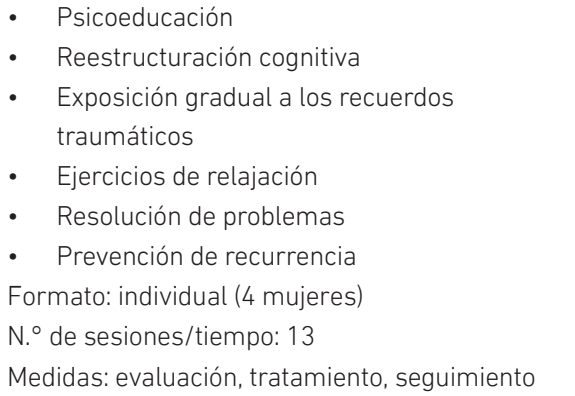 \\
\hline
\end{tabular}


(continuación)

\begin{tabular}{|c|c|c|}
\hline $\begin{array}{l}\text { Vaca-Ferrer et al. } \\
(2020)\end{array}$ & Terapias contextuales & $\begin{array}{l}\text { - Principios de la psicoterapia analítica } \\
\text { funcional (AF) } \\
\text { - Ejemplos y metáforas de la terapia de } \\
\text { aceptación y compromiso (ACT) } \\
\text { - Uso de recursos y tareas en la activación } \\
\text { conductual (AC) } \\
\text { Formato: grupal ( } 21 \text { mujeres) } \\
\text { N. }{ }^{\circ} \text { de sesiones/tiempo: } 11 \text { (120 min c/u) } \\
\text { Medidas: evaluación, tratamiento, postratamiento }\end{array}$ \\
\hline
\end{tabular}

Elaboración propia

En las intervenciones psicológicas para la violencia contra la mujer, predomina la TCC como propuesta de tratamiento. Sus fases son las siguientes: pretratamiento (evaluación), con el uso de pruebas psicológicas; tratamiento, con la ayuda de técnicas psicológicas; postratamiento y seguimiento en períodos variados desde un mes hasta un año. En cuanto a elementos similares, todas las intervenciones establecen la importancia de brindar psicoeducación como punto de partida, entrenamiento en control de la respiración, fortalecimiento de la autoestima, habilidades sociales, toma de decisiones, solución de problemas, técnicas cognitivas y de exposición.

Los programas de tratamiento planteados por Labrador y Rincón González (2002) como una intervención breve en formato grupal e individual son los más frecuentes. Están elaborados para disminuir los síntomas de TEPT desde la fase de evaluación, intervención y seguimiento respectivamente (Cáceres-Ortiz et al., 2011; Labrador y Alonso, 2007; Labrador et al., 2009). Cabe mencionar que gran parte de las propuestas de tratamiento fueron planteadas en formato grupal; sin embargo, la intervención fue aplicada en formato individual, en 8 a 20 sesiones, cada una con una duración de 50 a 120 minutos.

Otra característica del tratamiento a la mujer violentada es la existencia de políticas públicas de intervención, en las que encontró la limitación de tiempo y recursos económicos para el abordaje óptimo. Por otra parte, en órganos no gubernamentales, prevalece la recuperación del paciente (el tiempo que sea necesario). Los aspectos similares son la intervención en crisis si es necesario, la psicoeducación y la aplicación de terapia breve, donde se trabajan aspectos de empoderamiento y búsqueda de sistemas de apoyo para la mujer (Viñas Velásquez y Fernández de Juan, 2007).

Las propuestas más actuales dan una mirada distinta a la mujer maltratada, basadas en la intervención desde las terapias contextuales. Su objetivo es promover la validación, la aceptación y la activación conductual para que recuperen el control de sus vidas, actúen en una dirección acorde a los valores y se involucren en relaciones íntimas fundadas en la igualdad y el respeto (Vaca-Ferrer et al., 2020). 


\section{GRADO DE EFECTIVIDAD DE LOS TRATAMIENTOS PSICOLÓGICOS}

Se encontraron investigaciones que advierten la relevancia en efectividad de la TCC en la intervención para la mujer maltratada, donde se emplearon con mayor frecuencia técnicas de activación conductual, psicoeducación, técnicas de inervación vagal, manejo de estrés, resolución de problemas, respiración diafragmática, reestructuración cognitiva, técnicas de exposición, prevención de recaídas, entrenamiento asertivo, autoestima, autoeficacia, comunicación y habilidades sociales de acuerdo con las manifestaciones clínicas en estudio.

Cáceres-Ortiz et al. (2011) mostraron que en un $95 \%$ disminuyeron los síntomas de TEPT, ansiedad, depresión, autoestima, inadaptación, evitación (20,5 \% de las mujeres se quedaron con la sintomatología; sin embargo, lograron bajar en el seguimiento), hiperactivación (se redujo del $74 \%$ al 13,7 \% hasta el postratamiento) y las cogniciones postraumáticas. Estos resultados se mantuvieron durante el seguimiento tanto en el aspecto clínico como estadístico. También Santandreu y Ferrer-Pérez (2014) exponen evidencia de la reducción de la sintomatología de TEPT en un $85 \%$ a 3 meses de seguimiento, así como del $55 \%$ y el $75 \%$ de la depresión. Para Habigzang et al. (2018), estos síntomas de TEPT se mantuvieron estables, pero se consiguieron resultados estadísticamente significativos para los síntomas depresivos, ansiosos y estresores. De manera similar, Habigzang et al. (2019) revelan que 1 de 4 mantuvo los criterios diagnósticos de TEPT y permanecieron estables síntomas depresivos, pero hubo un impacto positivo en los niveles de ansiedad.

A la luz de estos resultados, en los programas desarrollados por Labrador y Rincón González, en formato grupal, se obtuvieron mejoras significativas para el $85 \%$ del TEPT; disminuyó la depresión al $2,9 \%$ al postratamiento y al $0 \%$ en el seguimiento; la baja autoestima y la ansiedad pasaron de $76,5 \%$ a $11,8 \%$ al postratamiento, y a $5 \%$ y $0 \%$ en el seguimiento al primer mes y a los tres meses. Estos resultados son respaldados por las percepciones de las participantes, quienes manifestaron sentirse mejor al finalizar el tratamiento (Labrador y Alonso, 2007). Mientras, en el formato individual, se alcanzó una mejora significativa en la sintomatología postraumática en más del 80 \% y en los problemas asociados: depresión, autoestima, inadaptación social y cogniciones postraumáticas. En el seguimiento a 3 y 6 meses, se encontró efectos estadísticamente significativos. También se consiguieron cambios clínicos en el 80 \% que no cumplió criterios diagnósticos hasta el postratamiento y seguimiento a los 6 meses. No obstante, no se halló una significación estadística en la mejora de la autoestima, pero el 62,5\% abandonó la categoría baja a los 6 meses de seguimiento (Labrador et al., 2009).

Asimismo, Matud et al. (2014), desde un formato individual, señalan una disminución estadísticamente significativa de la sintomatología depresiva (al 2,8 \% al seguimiento), ansiosa (al 2,8 \% al seguimiento) y de tipo postraumático (al 5,7 \% al seguimiento), así como de la inseguridad en sí mismas; a su vez, aumentó su autoestima, la confianza en 
sí mismas y disminuyó la inseguridad, lo que está respaldado por la significancia a nivel clínico. Por su parte, Madrigal et al. (2012), quienes emplearon el sistema inmunitario (inmunoglobulina A) en su estudio asociado a la sintomatología depresiva, ansiosa y de baja autoestima, alcanzaron una eficacia hacia el postratamiento (en comparación con el grupo de control) mostrando menor nivel de alteración psicológica y mayor nivel de inmunoglobulina $A$, por lo que es importante el tratamiento psicológico tanto en la salud física como emocional.

Sarasua et al. (2013), desde un programa individual cognitivo-conductual en mujeres víctimas de abuso sexual en la infancia, presentan en el postratamiento una tasa de éxito del $90,7 \%$ en cuanto al TEPT, del $64,5 \%$ en superación del malestar emocional (siendo clínicamente significativo) y del $81,2 \%$ en normalización de la conducta sexual. Un aspecto destacable es que las tasas de éxito terapéutico se mantienen relativamente constantes hasta el seguimiento de los 12 meses. Sin embargo, cabe destacar que hubo un nivel alto de rechazos y de descuido del tratamiento.

Iraizoz (2012) aborda las consecuencias psicológicas y psicopatológicas de la mujer maltratada desde la terapia racional emotiva (con técnicas cognitivas, conductuales y emocionales), en la que obtuvo resultados positivos en la reducción de frecuencia e intensidad de los síntomas psicopatológicos (TAG), como la depresión (de 22 a 0 puntos) y la ansiedad (de 77 a 10 puntos). Igualmente, un aumento considerable en el control de conductas de ira, puntuaciones bajas en la necesidad de dependencia, disminución de sentimientos de culpa, control de la ansiedad generalizada y las conductas agresivas mediante autoinstrucciones y el tiempo fuera. Este estudio plantea que la efectividad de las técnicas de TCC fue alta y el trabajo con las emociones ha sido complementario.

Un artículo particular entre los mencionados fue el de Jaramillo-Sierra y RipollNúñez (2018), quienes adaptaron y pusieron en marcha un programa de intervención para violencia situacional de parejas: Encontremos Soluciones Juntos (ESJ), donde participaron parejas que vivenciaban violencia. Los hallazgos evidenciaron que los participantes perciben cambios como la eliminación de la violencia física; la disminución de la violencia psicológica y frecuencia de los conflictos; el aumento de tiempo compartido, comunicación e intimidad de la pareja; el incremento de la capacidad de identificar signos de ira y detener a tiempo el escalamiento de dicha emoción.

Finalmente, con Vaca-Ferrer et al. (2020) nace la propuesta de un programa de intervención desde las terapias contextuales (técnicas de análisis funcional, terapia de aceptación y compromiso, activación conductual). El resultado demostró una disminución estadísticamente significativa en la severidad del malestar, en los indicadores de riesgo suicida y las secuelas de TEPT; también mejoraron las conductas problemáticas y la apertura hacia los demás. A un $23 \%$ se le redujeron los indicadores de malestar severo; de la misma forma, la satisfacción de las participantes tuvo puntajes altos (4/5). Por tanto, la intervención basada 
en la aceptación de los hechos y las emociones, así como trabajar con los valores y los objetivos de las participantes, es novedosa en este campo.

\section{TRATAMIENTO A LAS PRINCIPALES MANIFESTACIONES CLÍNICAS EN MUJERES VÍCTIMAS DE VIOLENCIA}

La violencia hacia la mujer deja secuelas psicológicas muy graves en las víctimas. Autores como Labrador et al. (2009), desde la aplicación de la terapia breve con el programa psicológico diseñado y validado por Labrador y Rincón González en el 2002, trabajan en el TEPT y problemas asociados: depresión, baja autoestima, inadaptación social y cogniciones postraumáticas. Este programa fue aplicado también por CáceresOrtiz et al. (2011), en formato individual, para tratar las mismas manifestaciones clínicas. En la misma línea, Habigzang et al. (2019) desde la TCC abordan el TEPT, ansiedad, depresión e inadaptación. Por su parte, Iraizoz (2012), quien utilizó la TCC, se enfoca en los TAG, trastornos de pánico, falta de control de impulsos (ira) y miedo a la separación producto de la violencia. Vaca-Ferrer et al. (2020), desde la terapia contextual, trató el suicidio, conductas problemáticas (agresión), dependencia emocional y sentimientos de culpa.

Finalmente, el TEPT, la depresión, la ansiedad, la dependencia emocional, la inadaptación y la baja autoestima son las principales consecuencias encontradas. Estos síntomas son abordados por los tratamientos antes descritos a fin de disminuirlos 0 eliminarlos, de manera que las víctimas se recuperen satisfactoriamente.

\section{CONCLUSIONES}

Esta revisión de literatura se centra en ayudar al profesional de la salud mental en el uso de técnicas que garanticen mayor efectividad en la intervención de las secuelas psicológicas y permitan una recuperación favorable que produzca un cambio a futuro de la vida de las mujeres víctimas de violencia conyugal.

El avance de tratamientos psicológicos en torno a este problema propuso la terapia cognitivo-conductual (TCC), la terapia racional emotiva (TRE) y las terapias contextuales. La TCC fue la más utilizada y coloca la dimensión cognitiva como un foco principal de cambio de otras áreas como la emoción y la conducta. En la aplicación de este modelo se presentan variaciones en los elementos o técnicas utilizadas, que dependen de los autores, la población en estudio y la sintomatología. Asimismo, destaca la significancia en efectividad de la TCC en los principales síntomas de la violencia contra la mujer, como el TEPT, la ansiedad, la depresión y la baja autoestima.

De igual manera, la propuesta de la TRE remarca la importancia de enfocar en el trabajo la cognición, siendo complementarias la emoción y la conducta. Por otro lado, 
desde las terapias contextuales se desarrolla una nueva línea de intervención que es igual de efectiva que la TCC, pero enfocada desde los "valores", "aceptación", etcétera, creando estrategias más integrales de intervención.

Es importante subrayar el establecimiento de líneas de tratamiento en organizaciones o centros gubernamentales para las mujeres que no cuentan con recursos económicos para pagar un tratamiento. Estas deben contar con un protocolo de abordaje centrado en las necesidades del paciente y que permita un espacio de empatía e interés en el caso. Así más mujeres tendrán la seguridad de que hay una salida al problema y podrán reincorporarse a la sociedad, de tal manera que construyan una vida saludable que valga la pena vivirla.

Por último, las limitaciones de este artículo no permiten llegar a una conclusión generalizable, por la falta de acceso a una cantidad más considerable de artículos, la búsqueda limitada al idioma español y el hecho de que el acceso a algunos artículos requería de pago. Superar estas limitaciones en trabajos próximos permitiría un enriquecimiento a este aporte. Sin embargo, las conclusiones encontradas nos satisfacen por dar una mirada más amplia y de opciones diversas a los distintos tratamientos para mujeres víctimas de maltrato, un problema que lleva consigo consecuencias no solo para las víctimas, sino también para su entorno, las cuales repercuten en su vida a largo plazo, por lo que se necesita aplicar una intervención más acertada.

\section{REFERENCIAS}

Bravo Campanón, C. (2008). Menores víctimas de violencia de género: experiencia de intervención en un centro de acogida para familias víctimas de violencia de género. Psychosocial Intervention, 17(3), 337-351.

Cáceres-Ortiz, E., Labrador-Encinas, F., Ardila-Mantilla, P., y Parada-Ortiz, D. (2011). Tratamiento cognitivo-conductual centrado en el trauma de mujeres víctimas de violencia de pareja. Psychologia. Avances de la Disciplina, 5(2), 13-31.

Calvo González, G., y Camacho Bejarano, R. (2014). La violencia de género: evolución, impacto y claves para su abordaje. Enfermería Global, 13(33), 424-439.

Habigzang, L. F., Gomes, F. P. M., y Maciel, Z. L. (2019). Terapia cognitivo-conductual para mujeres que sufrieron violencia por su pareja íntima. Estudio de casos múltiples. Ciencias Psicológicas, 13(2), 149-264.

Habigzang, L. F., Schneider, J. A., Petroli Frizzo, R., y Pizarro de Freitas, C. P. (2018). Evaluación del impacto de una intervención cognitivo-conductual para mujeres en situación de violencia doméstica en Brasil. Universitas Psychologica, 17(3), 1-11. 
Iraizoz, A. (2012). Violencia de género: tratamiento de un caso de trastorno de ansiedad generalizada. Acción Psicológica, 9(1), 115-122.

Jaramillo-Sierra, A. L., y Ripoll-Núñez, K. (2018). Adaptación de un programa de intervención para violencia situacional de parejas. Estudios Sociales, 66, 55-70.

Labrador, F. J., y Alonso, E. (2007). Eficacia a corto plazo de un programa de intervención para el trastorno de estrés postraumático en mujeres mexicanas víctimas de violencia doméstica. Psicopatología y Psicología Clínica, 12(2), 117-130.

Labrador, F. J., Fernández Velasco, R., y Rincón González, P. P. (2009). Evaluación de la eficacia de un tratamiento individual para mujeres víctimas de violencia de pareja con trastorno de estrés postraumático. Pensamiento Psicológico, 6(13), 49-67. Recuperado de https://www.redalyc.org/articulo.oa?id=80112469005

Labrador, F. J., y Rincón González, P. P. (2002). Trastorno de estrés postraumático en víctimas de maltrato doméstico: evaluación de un programa de intervención. Análisis y Modificación de Conducta, 28, 905-932.

Labrador, F. J., Vallejo, P. M., Matellanes, M. M., Echeburúa, O. R., Bados, L. A., y FernándezMontalvo, J. (2002). Eficacia de los tratamientos psicológicos. Recuperado de http://www.sepcys.es/uploads/documentos/Documento-Eficacia-Tratamientos -SEPCyS.pdf

Madrigal, L., Cardenal, V., Téllez, T., Ortiz-Tallo, M., y Jiménez, E. (2012). Cambios emocionales y alteraciones en la inmunoglobulina $\mathrm{A}(\lg \mathrm{A})$ tras el tratamiento psicológico en mujeres víctimas de violencia doméstica. Anales de Psicología, 28(2), 397-404.

Matud, P., Fortes, D., y Medina, L. (2014). Eficacia de un programa de tratamiento psicológico individual para mujeres maltratadas por su pareja. Psychosocial Intervention, 23, 199-207.

Organización Mundial de la Salud (OMS). (29 de noviembre del 2017). Violencia contra la mujer. Recuperado de https://www.who.int/es/news-room/fact-sheets/detail/ violence-against-women

Organización Panamericana de la Salud (OPS). (2018). La violencia contra la mujer afecta a casi el $60 \%$ de las mujeres en algunos países de las Américas. Recuperado de https: //www.paho.org/per/index.php?option=com_content\&view=article\&id=4190: la-violencia-contra-la-mujer-afecta-a-casi-el-60-de-las-mujeres-enalgunos-paises-de-las-americas\&ltemid=1062

Romero, I. (2010). Intervención en violencia de género. Consideraciones en torno al tratamiento. Psychosocial Intervention, 19(2), 191-199. 
Sarasua, B., Zubizarreta, I., De Corral, P., Echeburúa., E. (2013). Tratamiento psicológico de mujeres adultas víctimas de abuso sexual en la infancia: resultados a largo plazo. Anales de Psicología, 29(1), 29-37. http://dx.doi.org/10.6018/analesps.29.1.145281

Santandreu, M., y Ferrer-Pérez, V. A. (2014). Eficacia de un tratamiento cognitivo conductual para trastorno de estrés postraumático en víctimas de violencia de género. Psicología Conductual, 22(2), 239-256.

Vaca-Ferrer, R., Ferro-García, R., y Valero-Aguayo, L. (2020). Eficacia de un programa de intervención grupal con mujeres víctimas de violencia de género en el marco de las terapias contextuales. Anales de Psicología, 36(2), 188-199. https://doi. org/10.6018/analesps.396901

Viñas Velásquez, B. M., y Fernández de Juan, T. (2007). Atención psicológica que reciben mujeres víctimas de maltrato conyugal en organismos de Tijuana. Enseñanza e Investigación en Psicología, 12(2), 327-341. 